Proceedings of the 43rd "Jaszowiec", International School and Conference on the Physics of Semiconductors, Wisła 2014

\title{
ESR Spectroscopy of Graphene with Adsorbed NaCl Particles
}

\author{
E. KARPIERZ ${ }^{a, b}$, A. DrabińsKa ${ }^{a}$, R. BożEK ${ }^{a}$, P. KAŹMIERCZAK $^{a}$, A. WYSMOleK $^{a}$, \\ M. KAMIŃSKA ${ }^{a}$ AND W. STRUPIŃSKI ${ }^{c}$ \\ ${ }^{a}$ Faculty of Physics, University of Warsaw, L. Pasteura 5, 02-093 Warszawa, Poland \\ ${ }^{b}$ Faculty of Chemistry, Warsaw University of Technology, Warszawa, Poland \\ ${ }^{c}$ Institute of Electronic Materials Technology, Warszawa, Poland
}

Due to its peculiar properties graphene is a good candidate for sensor materials. Therefore, it is important to study influence of different fluids on graphene layer. The presented studies showed pinning of $\mathrm{NaCl}$ microcrystals to graphene surface after immersing graphene in $\mathrm{NaCl}$ solution and subsequent careful rinsing with distilled water. The atomic force microscopy images revealed presence of many NaCl-related structures over $100 \mathrm{~nm}$ high on graphene surface. The electron spin resonance spectrum for magnetic field perpendicular to the graphene layer consisted of several lines originating from $\mathrm{NaCl}$. The pinning of $\mathrm{NaCl}$ microcrystals resulted in increase of electron scattering, as confirmed by the Raman spectroscopy (the increase of intensity of $D$ and $D^{\prime}$ bands) and weak localization measurement (the decrease of coherence length).

DOI: $10.12693 /$ APhysPolA.126.1187

PACS: 81.05.ue, 72.10.Fk, 78.67.Wj, 72.80.Vp

\section{Introduction}

It is commonly expected that graphene will find a wide range of applications in many devices like ultrasensitive, label-free miniaturized electrostatic or electrochemical sensors [1]. Nanoelectronic graphene-based sensors and biosensors have already been used for detecting gas molecules, metal ions, various biomolecules and even metabolic activities of bacteria [2, 3]. Recently, graphene is considered to be applied for an active fluid flow sensor. Thinking about this application, besides measuring the influence of liquid flow rate on graphene electric response, it is very important to study the influence of different fluids on graphene layer properties.

In this paper we focus on atomic force microscopy (AFM), electron spin resonance (ESR), contactless electric transport and Raman spectroscopy techniques, to study the influence of exposure to $\mathrm{NaCl}$ solution on morphology and electron properties of graphene layer. Graphene samples were grown by chemical vapor deposition (CVD) method on copper foil and transferred onto $\mathrm{SiC}$ substrate. One of them was immersed in the $0.1 \mathrm{M} \mathrm{NaCl}$ solution. Then, after 3 days it was taken out and carefully rinsed with distilled water. The excess of water was removed by heating the sample at $150^{\circ} \mathrm{C}$. The sample treated this way will be called " $\mathrm{NaCl}$ treated graphene" further on, while as grown graphene in the same CVD process will be called a "reference graphene".

\section{Experiments and results}

The AFM method was used to examine the surface morphology of $\mathrm{NaCl}$ treated graphene and verify whether and how it differed from the reference graphene. Respective AFM images and height profiles are presented in Fig. 1. The surface morphology of the reference graphene was smoother and more homogeneous than that of $\mathrm{NaCl}$ treated one. Only a few structures up to approximately $50 \mathrm{~nm}$ height were visible, and the rest of the sample was flat with deviation not greater than $5 \mathrm{~nm}$. The surface of the $\mathrm{NaCl}$ treated graphene was much more rough and it had more structural defects, with appearance of excess structures about 3 times higher than that seen on the reference sample. It clearly confirmed the presence of $\mathrm{NaCl}$ particles on the surface, even though the sample had been carefully rinsed with distilled water.
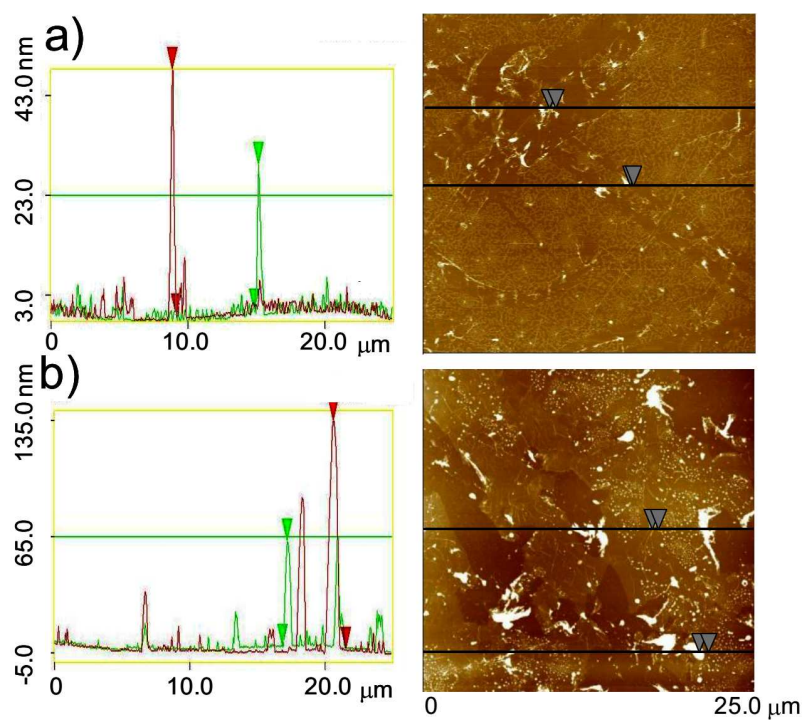

Fig. 1. AFM image of surface morphology for (a) reference graphene and (b) NaCl-treated graphene immersed. The height profiles are shown at the left side of each image.

The Raman spectroscopy was carried out using T64000 Horiba Jobin-Yvon spectrometer with Nd:YAG laser operating at $532 \mathrm{~nm}$ wavelength as an excitation source in the range between $1300 \mathrm{~cm}^{-1}$ and $2900 \mathrm{~cm}^{-1}$. This allowed to record graphene bands $\left(G, 2 D, D\right.$ and $\left.D^{\prime}\right)$ however did not allowed to record any bands from $\mathrm{NaCl}$ particles. The intensities of the $D$ and $D^{\prime}$ bands for $\mathrm{NaCl}$ treated graphene were higher in comparison with the reference one (Fig. 2). This proved the presence of additional short- and long-range scattering centers. The energy shifts and widths of $2 D$ and $G$ bands sug- 
gested strain relaxation in $\mathrm{NaCl}$ treated graphene [4]. Decrease of $G$-band to $D$-band peak intensity ratio $\left(I_{G} / I_{D}\right)$ of the Raman spectra indicated the increase of disorder in this sample. It was probably caused by exposing or enlarging the structural defects, present in as grown graphene. Grain diameter and distance between defects calculated from $I_{G} / I_{D}$ for the reference graphene were $L_{\alpha}=40.6 \mathrm{~nm}$ and $L_{D}=17.7 \mathrm{~nm}$, while for the $\mathrm{NaCl}$ treated graphene they were $L_{\alpha}=7.0 \mathrm{~nm}$ and $L_{D}=7.2 \mathrm{~nm}$, respectively $[5,6]$. The intensity ratio of $D$ and $D^{\prime}$ peak indicated the presence of vacancies and $s p^{3}$ hybridization defect type in $\mathrm{NaCl}$ sample [5].

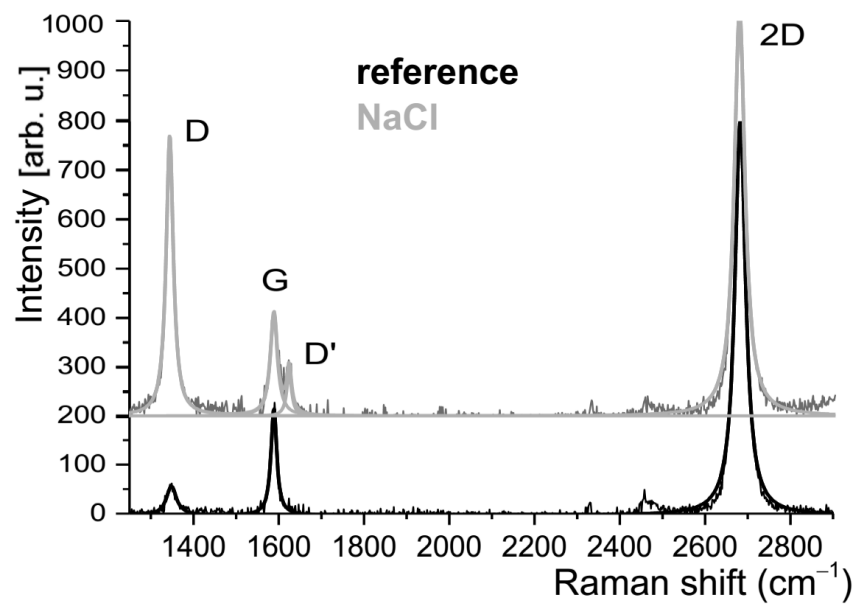

Fig. 2. Raman spectra of reference (black line) and $\mathrm{NaCl}$ treated graphene (grey line), obtained with $532 \mathrm{~nm}$ excitation from Nd:YAG laser.

ESR measurements were carried out using a Bruker ELEXSYS E580 CW ESR spectrometer, operating at microwave frequency $9.4 \mathrm{GHz}$ (X-band) and equipped with goniometer and helium cryostat allowing to reach temperatures down to $2 \mathrm{~K}$.

At $2 \mathrm{~K}$, for the reference graphene, one sharp resonance line was observed for magnetic field about $0.335 \mathrm{~T}$. This corresponded to $g=2.0027$ and evidently originated from $\mathrm{SiC}$ substrate (Fig. 3). For $\mathrm{NaCl}$ treated graphene several additional lines related to $\mathrm{NaCl}$ microcrystals were observed for magnetic field in the range between 0.31 and $0.37 \mathrm{~T}$, perpendicular to the sample surface (Fig. 3) [6]. The angular dependence of those lines showed the shift towards the higher magnetic field and rapid decrease of amplitude with deviation of angle from perpendicular orientation. It clearly indicated the formation and pinning of microcrystals on the graphene sample surface.

In the case of presence of electric field component of microwave in resonance cavity, the ESR spectrometer enables also measurement of the magnetoconductivity of the sample. The contactless method used in this paper is based on the measurements of $Q$ factor (quality factor) changes with the applied magnetic field $(\mathrm{d} Q / \mathrm{d} B)$ in the microwave-cavity of ESR spectrometer. The cavity $Q$ factor is changed due to either energy absorption for

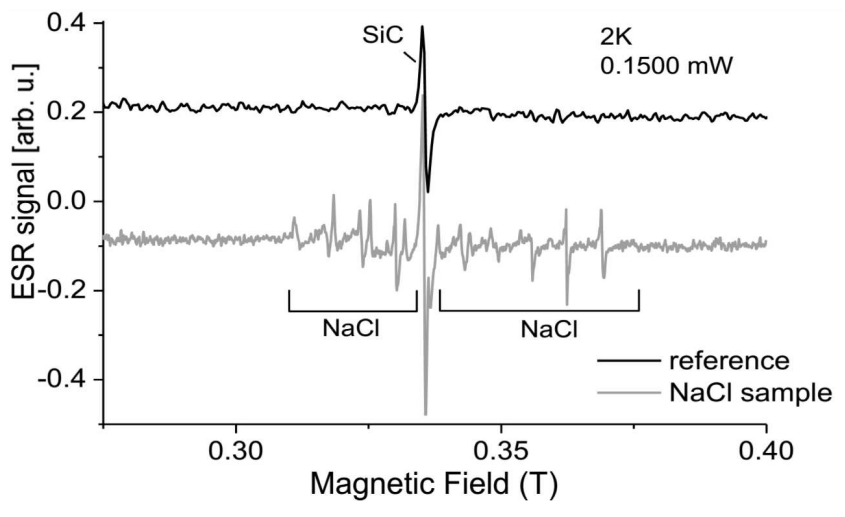

Fig. 3. ESR spectrum at $2 \mathrm{~K}$ for magnetic field perpendicular to the graphene layer of (a) reference graphene, (b) $\mathrm{NaCl}$ treated graphene.

electron spin transitions (classical ESR measurement) or induction of variation of the sample conductivity. In the second case, the signal recorded by the ESR spectrometer is $\mathrm{d} Q / \mathrm{d} B \propto \mathrm{d} \sigma / \mathrm{d} B$ where $\sigma$ is sample conductivity [7].

Therefore, in the ESR measurement it was possible to observe the magnetoconductance features like weak localization (WL) effects for graphene samples. The chirality of electrons in graphene with the Berry phase of $\pi$ suggests that the weak antilocalization effect should be expected in graphene. To explain the effect of restoring WL it is essential the elastic scattering changing the pseudospin and isospin. The trigonal warping breaks $p \rightarrow-p$ symmetry within each valley, which leads to the reductions of WL. On the other hand, it also lifts pseudospin symmetry, leading to modifications of WL. Changes in the substrate of epitaxial graphene give a smooth potential that conserves the pseudospin of chiral electrons, but is responsible for the change of isospin, point defects break the sublattice degeneracy locally, thereby causing intervalley scattering from one Dirac cone to the other. This reverses the electron phase and leads to the WL effect $[8,9]$.

The WL is associated with scattering mechanisms. At low temperature electrons moving in the diffusive way are scattered from potential disorder. The interference of electrons scattered in a closed loop and moving in opposite directions leads to enhancement of backscattering and reduction of conductivity of a sample, which is called WL. This effect is diminished in magnetic field resulting in positive magnetoconductance. Therefore the conductivity measurements can give information about electron coherence length, its mechanisms and elastic inter- and intravalley scatterings lengths.

The magnetic field dependence of conductance due to WL can be represented by equation $[10,11]$ :

$$
\begin{gathered}
\frac{\mathrm{d} \sigma(B)}{\mathrm{d} B}=\frac{\mathrm{e}^{2}}{\pi h}\left[\frac{1}{B_{\varphi}} F^{\prime}\left(\frac{B}{B_{\varphi}}\right)-\frac{1}{B_{\varphi}+2 B_{\mathrm{i}}} F^{\prime}\left(\frac{B}{B_{\varphi}+2 B_{\mathrm{i}}}\right)\right. \\
\left.-\frac{2}{B_{\varphi}+B_{\mathrm{i}}+B_{\mathrm{lr}}} F^{\prime}\left(\frac{B}{B_{\varphi}+B_{\mathrm{i}}+B_{\mathrm{lr}}}\right)\right],
\end{gathered}
$$


where $B_{\varphi, i, \mathrm{lr}}=\hbar /\left(4 e L_{\varphi, i, \mathrm{lr}}^{2}\right)$ are proportional to the decoherence and scattering rates, $L_{\varphi}$ is coherence length, $L_{\mathrm{lr}}$ - long range elastic scattering length due to intravalley scattering and warping, $L_{\mathrm{i}}$ - intervalley elastic scattering length and $F^{\prime}(z)=1 / z-\Psi^{(1)}(1 / 2+1 / z) / z^{2}$, and $\Psi^{(1)}$ — trigamma function.

For both samples for zero magnetic field, $\mathrm{d} \sigma / \mathrm{d} B$ equals zero and changes its sign from negative to positive. This corresponds to the minimum conductance value and positive magnetoconductance.

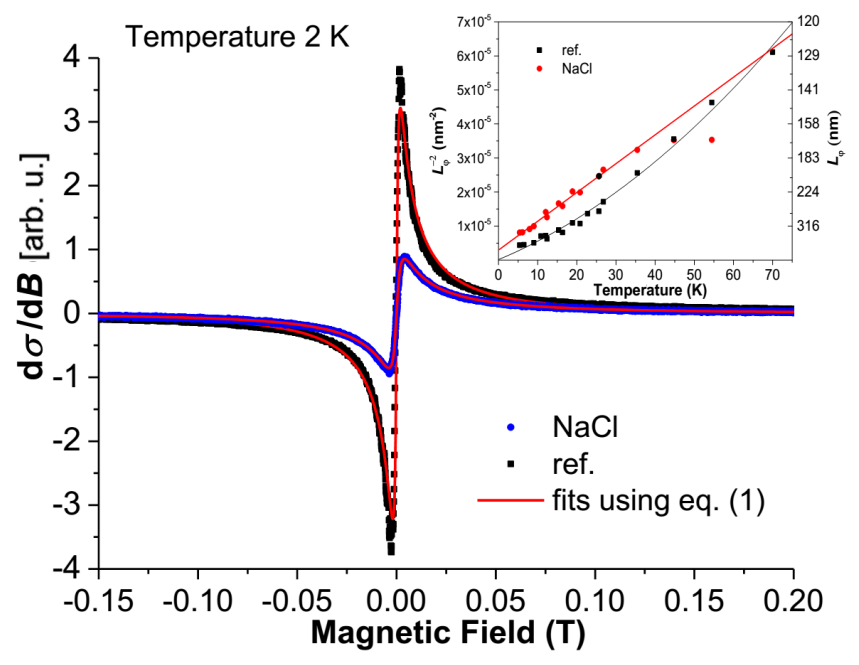

Fig. 4. The signal measured by an ESR spectrometer, proportional to $\mathrm{d} \sigma / \mathrm{d} B$ at $2 \mathrm{~K}$ (magnetic field perpendicular to graphene sheets) and temperature dependence of the coherence length $\left(L_{\varphi}\right)$ for $\mathrm{NaCl}$ treated graphene (red points) and reference graphene (black points) (inset). The elastic scattering lengths are: for $\mathrm{NaCl}$ treated graphene $L_{\mathrm{i}}=117 \mathrm{~nm}, L_{\mathrm{lr}}=21 \mathrm{~nm}$, for reference one: $L_{\mathrm{i}}=127 \mathrm{~nm}, L_{\mathrm{lr}}=13 \mathrm{~nm}$.

For $\mathrm{NaCl}$ treated graphene the amplitude of WL signal decreased, while its width increased when comparing to the reference graphene (Fig. 4). The fit of the signal with Eq. (1) gave the temperature independent values of elastic scattering lengths, which occurred very similar for both samples. The difference was in inelastic scatterings. The linear dependence of $L_{\varphi}^{-2}(T)$ for $\mathrm{NaCl}$ treated graphene indicated the electron-electron scattering in a diffusive regime as a main inelastic scattering mechanism [7, 8]. Instead, the square dependence of $L_{\varphi}^{-2}(T)$ for reference sample proved the ballistic regime of electron-electron scattering [12]. Non-zero offset of $L_{\varphi}^{-2}(T)$ for $\mathrm{NaCl}$ treated graphene indicated appearance of temperature independent inelastic scattering, reducing the coherence length at $0 \mathrm{~K}$ to $219 \mathrm{~nm}$.

\section{Conclusions}

We presented results of AFM, Raman and ESR spectroscopies, and contactless studies of weak localization of epitaxial graphene sheets on $\mathrm{SiC}$ substrate immersed in $0.1 \mathrm{M} \mathrm{NaCl}$ solution, in comparison with the reference as grown graphene. AFM images of both samples revealed the difference in surface morphology. The reference graphene was more homogeneous and flat than the
$\mathrm{NaCl}$ treated graphene, for which the structures present on the surface were higher and the surface itself was distinctly rough. This clearly confirmed the presence of the $\mathrm{NaCl}$ nanocrystals on graphene after immersion in $\mathrm{NaCl}$ solution, even though the careful rinsing with distilled water was performed. In the ESR spectrum sharp resonance line originating from $\mathrm{SiC}$ substrate and additionally, for $\mathrm{NaCl}$ treated graphene, several ESR lines originating from $\mathrm{NaCl}$ microcrystals were seen. They were clearly visible for magnetic field perpendicular to the graphene layer. The Raman spectrum of $\mathrm{NaCl}$ treated graphene showed the increase of $D$ and $D^{\prime}$ bands intensities. This proved the additional short- and long-range scattering introduced by immersion in $\mathrm{NaCl}$ solution. The energy shifts and the widths of $2 D$ and $G$ bands suggested strain relaxation in graphene layer. The analysis of the coherence length $\left(L_{\varphi}\right)$ indicated the presence of temperature independent inelastic scattering for $\mathrm{NaCl}$ treated graphene, reducing the coherence length at $0 \mathrm{~K}$, and electron-electron scattering in a diffusive regime as a main inelastic scattering mechanism.

\section{Acknowledgments}

This work was partially supported by the NCN grant no 2012/07/B/ST3/03220, Poland and NCBiR grant GRAF-TECH/NCBR/02/19/2012, Poland.

\section{References}

[1] W. Yuan, G. Shi, J. Mater. Chem. A 1, 10078 (2013).

[2] J.D. Fowler, M.J. Allen, V.C. Tung, Y. Yang, R.B. Kaner, B.H. Weiller, ACS Nano 3, 301 (2009).

[3] F. Schedin, A.K. Geim, S.V. Morozov, E.W. Hill, P. Blake, M.I. Katsnelson, K.S. Novoselov, Nature Mater. 6, 652 (2007).

[4] I. Childres, L.A. Jauregui, W. Park, H. Cao, Y.P. Chen, in: Developments in Photon and Materials Research, Ed. J.I. Jang, Nova Sci. Publ., 2013.

[5] F. Tuinstra, L.J. Koenig, J. Chem. Phys. 53, 1126 (1970).

[6] L.G. Cançado, A. Jorio, E.H. Martins Ferreira, F. Stavale, C.A. Achete, R.B. Capaz, M.V.O. Moutinho, A. Lombardo, T.S. Kulmala, A.C. Ferrari, Nano Lett. 11, 8 (2011).

[7] B.L. Altshuler, A.G. Aronov, D.E. Khmelnitsky, J. Phys. C 15, 7367 (1982).

[8] E. McCann, K. Kechedzhi, V.I. Fal'ko, H. Suuzuura, T. Ando, B.L. Altshuler, Phys. Rev. Lett. 97, 146805 (2006).

[9] A. Drabińska, M. Kamińska, A. Wołoś, W. Strupiński, A. Wysmołek, W. Bardyszewski, R. Bożek, J.M. Baranowski, Phys. Rev. B 88, 165413 (2013).

[10] A. Eckmann, A. Felten, A. Mishchenko, L. Britnell, R. Krupke, K.S. Novoselov, C. Casiraghi, Nano Lett. 12, 3925 (2012).

[11] M. Ikeya, J.H. Crawford Jr., Phys. Status Solidi B 58, 643 (1973).

[12] A. Drabińska, A. Wołoś, M. Kamińska, W. Strupinski, J.M. Baranowski, Phys. Rev. B 86, 045421 (2012). 